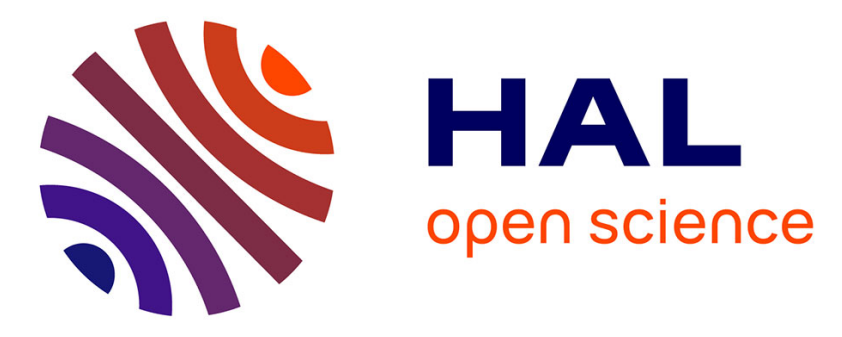

\title{
Effets d'une alcalose induite sur les paramètres métaboliques et sanguins lors d'un test
}

\author{
Christophe Hausswirth, François Bieuzen, Sébastien Argentin, Jean-Michel \\ Lévêque, Estelle Patou
}

\section{- To cite this version:}

Christophe Hausswirth, François Bieuzen, Sébastien Argentin, Jean-Michel Lévêque, Estelle Patou. Effets d'une alcalose induite sur les paramètres métaboliques et sanguins lors d'un test: Cyclisme et Course à pied chez des triathlètes entraînés. Science \& motricité: Revue scientifique de l'Association des Chercheurs en Activités Physiques et Sportives, 2004, 1 (51), pp.85 - 101. 10.3917/sm.051.0085 . hal-01765328

\section{HAL Id: hal-01765328 \\ https: / hal-insep.archives-ouvertes.fr/hal-01765328}

Submitted on 12 Apr 2018

HAL is a multi-disciplinary open access archive for the deposit and dissemination of scientific research documents, whether they are published or not. The documents may come from teaching and research institutions in France or abroad, or from public or private research centers.
L'archive ouverte pluridisciplinaire HAL, est destinée au dépôt et à la diffusion de documents scientifiques de niveau recherche, publiés ou non, émanant des établissements d'enseignement et de recherche français ou étrangers, des laboratoires publics ou privés. 


\section{EFFETS D'UNE ALCALOSE INDUITE SUR LES PARAMÈTRES MÉTABOLIQUES ET SANGUINS LORS D'UN TEST}

Cyclisme et Course à pied chez des triathlètes entraînés

Christophe Hausswirth et al.

EDP Sciences | Movement \& Sport Sciences

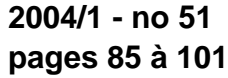

2004/1 - no 51

pages 85 à 101

ISSN 1378-1863

Article disponible en ligne à l'adresse:

http://www.cairn.info/revue-science-et-motricite-2004-1-page-85.htm

Pour citer cet article :

Hausswirth Christopheet al., « Effets d'une alcalose induite sur les paramètres métaboliques et sanguins lors d'un test » Cyclisme et Course à pied chez des triathlètes entraînés,

Movement \& Sport Sciences, 2004/1 no 51, p. 85-101. DOI : 10.3917/sm.051.0085

Distribution électronique Cairn.info pour EDP Sciences.

(c) EDP Sciences. Tous droits réservés pour tous pays.

La reproduction ou représentation de cet article, notamment par photocopie, n'est autorisée que dans les limites des conditions générales d'utilisation du site ou, le cas échéant, des conditions générales de la licence souscrite par votre établissement. Toute autre reproduction ou représentation, en tout ou partie, sous quelque forme et de quelque manière que ce soit, est interdite sauf accord préalable et écrit de l'éditeur, en dehors des cas prévus par la législation en vigueur en France. II est précisé que son stockage dans une base de données est également interdit. 


\title{
Effets d'une alcalose induite sur les paramètres métaboliques et sanguins lors d'un test Cyclisme - Course à pied chez des triathlètes entraînés
}

\author{
Christophe Hausswirth, François Bieuzen, Sébastien Argentin, \\ Jean-Michel Levêque ${ }^{(1)}$ et Estelle Patou ${ }^{(2)}$
}

\section{RÉSUMÉ}

L'objectif de ce travail était d'étudier l'effet d'une ingestion de boisson alcalinisante sur l'évolution des valeurs de consommation maximale d'oxygène ( $\dot{\mathrm{V}} \mathrm{O}_{2}$ max) en cyclisme, ainsi que sur les valeurs métaboliques et sanguines lors d'un temps limite en course à pied réalisé en enchaînement. Neuf triathlètes entraînés ont réalisé quatre tests d'enchaînement Ergocycle-Tapis roulant, suivant une modalité triangulaire en cyclisme (i.e. $\dot{V} \mathrm{O}_{2}$ max) et rectangulaire en course à pied ( $T_{\text {lim }}$ ) après une minute de repos entre les deux tests. Ils ont ingéré, après tirage au sort, 1h30 avant le test une solution de Citrate de sodium tri-sodique (C) à $0,3 \mathrm{~g} . \mathrm{kg}^{-1}$ de poids corporel, d'hydrate de carbone avec vitamines et minéraux (CHO), d'un Placebo $(P)$ et d'une solution mélangeant Citrate de sodium et $\mathrm{CHO}(\mathrm{C}-\mathrm{CHO})$. Les valeurs $\dot{V} \mathrm{O}_{2}$ max n'étaient pas modifiées sur ergocycle. La puissance maximale aérobie (Pmax) était augmentée $(p<0,05)$ de $3 \%$ en $\mathrm{C}-\mathrm{CHO}$ vs. $\mathrm{C}$, de $6 \%(p<0,05)$ en $\mathrm{C}-\mathrm{CHO}$ vs. $\mathrm{CHO}$, et de $8 \%(p<0,05)$ en $\mathrm{C}-\mathrm{CHO}$ vs. $\mathrm{P}$. Les résultats de $\mathrm{T}_{\text {lim }}$ montrent une amélioration de $8 \%$ $(p<0,05)$ en $\mathrm{C}-\mathrm{CHO}$ vs. $\mathrm{C}$, de $14 \%(p<0,01)$ en $\mathrm{C}-\mathrm{CHO}$ vs. $\mathrm{CHO}$, et de $21 \%(p<0,01)$ en $\mathrm{C}-\mathrm{CHO}$ vs. P. Ces valeurs étaient reliées au \% de $\dot{\mathrm{V}} \mathrm{O}_{2}$ max utilisés lors du test. Les valeurs de $\mathrm{pH}$, de bicarbonates et de lactates sanguins étaient plus élevées après le test d'ergocycle et le $\mathrm{T}_{\text {lim }}$ en $\mathrm{C}$ et $\mathrm{C}-\mathrm{CHO}$, comparées à $\mathrm{P}$ et $\mathrm{CHO}(p<0,05)$. La solution de $\mathrm{C}-\mathrm{CHO}$ apparaît reliée aux effets isolés du citrate ingéré seul, avec toutefois de meilleures Pmax en cyclisme et une augmentation des $T_{\text {lim }}$ en course à pied : ces résultats semblent davantage reliés à des paramètres métaboliques et d'efforts perçus et peu corrélés aux variations des gaz du sang et de l'alcalose induite.

Mots-clés : Triathlètes, $\mathrm{V}_{2} \max$, temps limite, lactatémie, citrate de sodium, statut acido-basique

(1) Laboratoire de biomécanique et physiologie, INSEP, 75012 PARIS

(2) Fédération Française de Triathlon 


\title{
Effects of an induced alkalosis on metabolic and blood parameters using a Cycling-Running test in trained triathletes
}

\begin{abstract}
The aim of the present study was to compare the effect of an alkalosis drink ingestion on the oxygen uptake $\left(\mathrm{VO}_{2}\right.$ max) values in biking, and on blood and metabolic values during a $\mathrm{T}_{\text {lim }}$ running test done in transition. Nine trained triathletes realised 4 cycle-run tests, where the cycling test was a $\dot{V} \mathrm{O}_{2}$ max test and the running test was a $T_{\text {lim }}$ on a treadmill at ventilatory threshold. They ingested in a random order 1 h30 before the cycle-run test either a solution of sodium citrate (C) at $0,3 \mathrm{~g}_{ } \mathrm{kg}^{-1}$ body weight, either a solution of carbohydrates associated with vitamins and minerals (CHO), either a placebo $(P)$ and either a mixed drink of sodium citrate and $\mathrm{CHO}(\mathrm{C}-\mathrm{CHO}) . \dot{\mathrm{V}} \mathrm{O}_{2}$ max are similar in cycling. Maximal aerobic power (Pmax) increased $(p<0,05)$ in $3 \%$ in $\mathrm{C}-\mathrm{CHO}$ vs. $\mathrm{C}$, in $6 \%(p<0,05)$ in $\mathrm{C}-\mathrm{CHO}$ vs. $\mathrm{CHO}$, and in $8 \%(p<0,05)$ in $\mathrm{C}-\mathrm{CHO}$ vs. $\mathrm{P} . \mathrm{T}_{\text {lim }}$ results showed an increase in $8 \%(p<0,05)$ in $\mathrm{C}-\mathrm{CHO}$ vs. C, $14 \%(p<0,01)$ in $\mathrm{C}-\mathrm{CHO}$ vs. $\mathrm{CHO}$, and $21 \%(p<0,01)$ in $\mathrm{C}-\mathrm{CHO}$ vs. P. These values were linked to the \% of $\dot{V}_{2}$ max used during the test. Moreover, blood $p H$, blood bicarbonates and lactates values were higher after cycling and the $T_{\text {lim }}$ running test in $C$ and $C$ $\mathrm{CHO}$, compared to $\mathrm{P}$ et $\mathrm{CHO}(p<0,05)$. The $\mathrm{C}-\mathrm{CHO}$ drink seemed linked to the results obtained in sodium citrate isolated drink, but with however best Pmax in cycling and a significant increase in $T_{\text {lim }}$ during running : these results are more related to the modifications of metabolic parameters and ratings of perceived exertion, and less correlated to the variations of blood gaz values and the induced alkalosis.
\end{abstract}

Key-words: Triathletes, $\dot{\mathrm{V}} \mathrm{O}_{2} \max$, Time limit, Lactatemia, Sodium citrate, Acid-base status

\section{Introduction}

L'aptitude physique d'un individu comprend plusieurs composantes ; dans ce cadre, de nombreuses études ont pu montrer que la consommation maximale d'oxygène $\left(\mathrm{VO}_{2} \mathrm{max}\right)$ est un bon indice de l'aptitude bioénergétique aux exercices sollicitant prioritairement la source d'énergie aérobie. Cependant, il est bien établi qu'aucune variable physiologique isolée ne peut être un indicateur unique associé à la performance réalisée au cours de la réalisation de sports à forte demande énergétique comme le cyclisme ou la course à pied (Hausswirth \& Brisswalter, 1999). Avec l'apparition de sports d'endurance relativement récents comme le 
triathlon (natation, cyclisme, course à pied), le coureur est confronté à de nouvelles sensations lorsqu'il aborde la dernière épreuve. À ce jour, aucun test de routine en terme d'évaluation physiologique maximale n'a pu être mis en évidence chez les triathlètes entraînés afin préciser la particularité réelle de l'enchaînement ergocycle — tapis roulant en laboratoire.

Il est bien documenté (Sahlin 1978) que lors d'un exercice court et intense ou à l'issue d'un test de $\mathrm{VO}_{2}$ max, la production d'acide lactique dans les muscles actifs et sa diffusion rapide dans les liquides extracellulaires entraînent une élévation des concentrations d'ions hydrogènes. De nombreuses observations (Hermansen \& Osnes, 1972 ; Sahlin 1978) ont conduit à l'hypothèse qu'au cours d'un exercice maximal ou supramaximal, l'acidose métabolique pourrait être un facteur responsable de l'apparition de la fatigue musculaire. À partir de ces observations, plusieurs travaux scientifiques (Kowalchuk et coll., 1984 ; Jones et coll., 1977) ont cherché à déterminer dans quelle mesure un apport en substances tampons (type bicarbonates) ingéré avant un effort pouvait améliorer la performance, en retardant la baisse du $\mathrm{pH}$ intramusculaire, et en permettant une plus grande production d'ions lactates. Les résultats de ces études fournissent des observations contradictoires. En effet, une amélioration de la performance associée à une plus grande élévation de la lactatémie après ingestion a été rapportée par Jones et coll. (1977) au cours d'un exercice maximal à charge graduellement augmentée, ou au cours d'un temps limite isométrique mené jusqu'à épuisement lors d'un exercice d'extension du genou (Hausswirth et coll., 1995). À l'inverse, d'autres travaux (Kinderman et coll., 1977) n'ont observé aucun effet significatif au cours d'exercices supra-maximaux compris entre 30 et 60 secondes.

D'un point de vue physiologique, le déséquilibre acido-basique sanguin perturbe à la fois le système endocrinien, la disponibilité en substrats sanguins et musculaires (Sutton et coll., 1981), les systèmes cardio-circulatoire et ventilatoire (Jones et coll., 1977) ainsi que la perception de l'effort (Swank \& Robertson, 1989). Si l'importance de l'ingestion de bicarbonate de sodium $\left(\mathrm{NaHCO}_{3}\right)$ a été largement utilisée à fortes doses ( 0,3 à 0,5 g. $\mathrm{kg}^{-1}$ poids corporel) pour mettre en évidence une amélioration des performances en course à pied (Wilkes et coll., 1983), cellesci sont souvent associées à des troubles gastro-intestinaux (voir revue : Linderman \& Gosselink, 1994). Une autre substance a reçu l'attention des chercheurs ces dernières années, il s'agit du citrate de sodium trisodique $\left(\mathrm{Na}_{3} \mathrm{C}_{6} \mathrm{H}_{5} \mathrm{O}_{7}\right)$ : son effet ergogénique a été montré dans quelques 
études (McNaughton 1990 ; Hausswirth et coll., 1995) sans pour autant être associé ou comparé à des hydrates de carbone ingérés avant un exercice mené jusqu'à épuisement.

Les buts de notre étude étaient :

1. De déterminer l'influence de l'ingestion pré-exercice de Citrate de Sodium Tri-sodique (C) sur les valeurs de $\mathrm{VO}_{2}$ max en cyclisme et de temps limite (Tlim) en course à pied, en comparaison avec un groupe placebo $(\mathrm{P})$,

2. De comparer les résultats sanguins et métaboliques obtenus entre les situations d'alcalose induite seule (C), d'alcalose induite associée à des hydrates de carbone avec vitamines et minéraux (C$\mathrm{CHO})$, la situation placebo $(\mathrm{P})$ et la situation d'hydrates de carbone avec vitamines et minéraux seule $(\mathrm{CHO})$, et de les relier à la performance et aux variations d'efforts perçus.

L'hypothèse de notre travail était de montrer qu'une ingestion préexercice d'une boisson énergétique alcalinisante devait autoriser des performances plus élevées $\left(\mathrm{VO}_{2} \mathrm{max}, \mathrm{T}_{\text {lim }}\right)$ dans un test spécifique d'évaluation physiologique mis au point chez les triathlètes entraînés.

\section{Matériels et méthodes}

\section{Sujets}

Les sujets de cette expérimentation étaient au nombre de neuf, de sexe masculin (âge: 21,1 $\pm 3,8$ ans ; masse : 68,1 $\pm 5,8 \mathrm{~kg}$; taille : 177,5 $\pm 3,4 \mathrm{~cm} ; c f$. Tableau 1), tous triathlètes et/ou duathlètes bien entraînés (entraînement hebdomadaire $: 22 \mathrm{~h} \pm 4$ ). Leur meilleure performance de l'année sur un triathlon distance olympique était de $1 \mathrm{~h} 58 \mathrm{~min} \pm 4 \mathrm{~min}$, et était habitué à courir sur tapis roulant. La pratique de l'enchaînement vélo-course à pied était également habituelle à l'entraînement. Afin de participer à cette étude, ils ont été informés de la nature des possibles problèmes associés à l'expérimentation. Tous les sujets étaient volontaires et motivés par la réalisation de l'expérimentation. La présente étude a été approuvée par le comité d'éthique de la protection des personnes de Paris-Cochin. 


\begin{tabular}{|c|c|c|c|c|c|c|c|c|c|}
\hline $\begin{array}{l}\text { Age } \\
\text { (ans) }\end{array}$ & $\begin{array}{c}\text { Masse } \\
(\mathrm{kg})\end{array}$ & $\begin{array}{l}\text { Taille } \\
\text { (cm) }\end{array}$ & $\begin{array}{c}\text { FCmax } \\
\text { (batt. } \\
\text { min }^{-1} \text { ) }\end{array}$ & $\begin{array}{c}\dot{\mathrm{V}} \mathrm{O}_{2} \max \\
\left(\mathrm{mL}^{\mathrm{kg}} \mathrm{kg}^{1}\right. \\
\left.\min ^{-1}\right)\end{array}$ & $\begin{array}{l}{\left[\mathrm{La}^{-}\right]} \\
(\mathrm{mM})\end{array}$ & $\begin{array}{c}\text { \% de } \\
\dot{\text { VO }}{ }_{2} \max \\
\text { à } S_{\mathrm{V} 2}\end{array}$ & $\begin{array}{c}\text { VMA } \\
\left(\mathrm{km}^{\left.-\mathrm{h}^{-1}\right)}\right.\end{array}$ & $\begin{array}{c}\% \\
\text { masse } \\
\text { grasse }\end{array}$ & $\begin{array}{l}\text { Entraîn. } \\
\text { Hebdo. } \\
\text { (heures) }\end{array}$ \\
\hline $\begin{array}{l}24,1 \\
\pm 3,8\end{array}$ & $\begin{array}{c}68,1 \pm \\
5,8\end{array}$ & $\begin{array}{l}177,5 \\
\pm 3,4\end{array}$ & $191 \pm 7$ & $69,2 \pm 5,1$ & $\begin{array}{c}10,2 \pm \\
1,4\end{array}$ & $79,2 \pm 6,1$ & $20,3 \pm 0,6$ & $\begin{array}{c}9,2 \pm \\
1,2\end{array}$ & $22 \pm 4$ \\
\hline
\end{tabular}

TABLEAU I

Caractéristiques descriptives des sujets associées aux valeurs obtenues lors du test triangulaire effectué sur tapis roulant ( $0 \%$ de pente). Les valeurs sont moyennées $\pm E T$.

$\mathrm{FCmax}=$ Fréquence Cardiaque maximale

$\dot{\mathrm{V}} \mathrm{O}_{2} \max =$ consommation maximale d'oxygène

[La- $]$ = lactatémie de fin de test

$\mathrm{S}_{\mathrm{V} 2}=$ second seuil ventilatoire

$\mathrm{VMA}=$ Vitesse Maximale Aérobie

\section{Test de consommation maximale d'oxygène sur tapis roulant}

Les neuf sujets ont réalisé une épreuve maximale de détermination de la vitesse maximale aérobie (VMA) sur tapis roulant (Gymrol, France) placé volontairement à $0 \%$ de pente. Au cours de ces tests, les paramètres gazeux et respiratoires étaient enregistrés pendant toute la durée des tests au moyen d'un système télémétrique de type Cosmed $K 4_{\mathrm{RQ}}{ }^{(0)}$ (Rome, Italie) validé scientifiquement (Hausswirth, Bigard, \& Le Chevalier, 1997). La consommation d'oxygène $\left(\mathrm{VO}_{2}\right)$, le débit ventilatoire $\left(\mathrm{V}_{\mathrm{E}}\right)$ et la fréquence respiratoire (FR) ont été les principaux paramètres pris en considération ( $c f$. Tableau 1). Après un échauffement de 6 minutes à $10 \mathrm{~km} \cdot \mathrm{h}^{-1}$, l'épreuve débutait à $11 \mathrm{~km} \cdot \mathrm{h}^{-1}$ puis était incrémentée de $1 \mathrm{~km} . \mathrm{h}^{-1}$ toutes les minutes. Nous avons pu vérifier pour chaque individu l'atteinte des critères de $\mathrm{VO}_{2}$ max proposés par Howley et coll. (1995), i.e. une atteinte d'un plateau de $\mathrm{VO}_{2}$ malgré une augmentation de l'intensité de l'exercice, une fréquence cardiaque correspondant à 90 \% de la fréquence cardiaque maximale théorique, un quotient respiratoire supérieur à 1.15, et une lactatémie post-exercice supérieure ou égale à $8 \mathrm{mM}$. La lactatémie pré, per et post-exercice a été analysée à partir de l'appareil Lactate Pro ${ }^{\circledR}$ (Arkray — Monitor, Anglet, France). La vitesse maximale aérobie était la vitesse la plus élevée complètement réalisée par les sujets. Le seuil ventilatoire $\left(\mathrm{SV}_{2}\right)$ a été obtenu d'après la méthode de calcul proposée par Beaver, Wasserman, \& Whipp (1986), 
méthode basée sur la recherche systématique du point de rupture de la relation $\mathrm{VCO}_{2} / \mathrm{VO}_{2}$. Tous les tests ont été réalisés aux mêmes instants de la journée, après un repas standardisé. Les sujets n'ont pas effectué d'entraînement épuisant dans les deux jours précédant le test.

\section{Test d'enchaînement Ergocycle-Tapis roulant (E-TR)}

\section{Protocole}

Après $48 \mathrm{~h}$ de repos ou d'activité physique modérée, les neuf sujets ont été soumis à un test triangulaire mené jusqu'à épuisement sur ergocyle (Lode ${ }^{\odot}$ - Type Excalibur, Groningen, Les Pays-bas). Le protocole débutait par 6 minutes d'échauffement à 100 watts, puis la puissance était incrémentée de 25 watts toutes les 2 minutes jusqu'à l'atteinte des critères de $\mathrm{VO}_{2} \max$ (Howley et coll., 1995). Après une minute de récupération pendant laquelle les triathlètes changeaient de chaussures et allaient sur le tapis roulant, ils débutèrent le $\mathrm{T}_{\text {lim }}$ en course à pied à la vitesse de $\mathrm{SV}_{2}$ calculée lors du test triangulaire sur tapis roulant. Cette vitesse a été démontrée comme étant proche de celle obtenue lors du $10 \mathrm{~km}$ de course à pied lors d'un triathlon de distance olympique (Hausswirth et coll., 1996).

\section{Sessions expérimentales et constitution des boissons ingérées}

$1 \mathrm{~h} 30$ avant le test E-TR, chaque sujet devait ingérer une des quatre solutions proposées avec tirage au sort préalable :

1. Citrate de Sodium Tri-sodique (C), contenant $0,3 \mathrm{~g}$ de $\mathrm{Na}_{3} \mathrm{C}_{6} \mathrm{H}_{5} \mathrm{O}_{7}$ par $\mathrm{kg}$ de poids corporel dilués dans 1 litre d'eau,

2. Placebo (P), contenant du chlorure de sodium dosé à $0,045 \mathrm{~g} \cdot \mathrm{kg}^{-1} \mathrm{de}$ poids corporel,

3. Hydrates de carbone - vitamines - minéraux (CHO), contenant $5,5 \%$ de $\mathrm{CHO}$ (fructose, maltodextrine) soit $55 \mathrm{~g} . \mathrm{L}^{-1}$, et des vitamines (A, C, B1, B6), du $\beta$ carotène, des bioflavonoïdes d'agrumes, et des minéraux (magnésium et calcium),

4. Citrate de sodium tri-sodique + Hydrates de carbone - vitamines - minéraux (C-CHO), contenant la solution $1(\mathrm{C})+$ la solution 3 $(\mathrm{CHO})$.

Tous les sujets ont passé les 4 situations, ingérant par là-même les 4 boissons au hasard 1 h30 avant la réalisation du test $\mathrm{E}-\mathrm{TR}$. Le régime alimentaire était contrôlé et identique $48 \mathrm{~h}$ avant la passation d'un test. 
De plus, $72 \mathrm{~h}$ de récupération étaient données aux sujets entre chaque test E-TR.

\section{Prélèvements sanguins}

Les prélèvements sanguins ont été réalisés au lobule de l'oreille dilaté au préalable pendant 5 minutes avec une vasodilatateur local (Finalgon, Düsseldorf, Allemagne). Une quantité de $100 \mu$ l de sang fut prélevée à chaque prise au moyen d'un capillaire pré-hépariné et placé immédiatement dans un appareil de mesure de gazométrie sanguine (i-STAT corporation $^{\circledR}$, New-Jersey, Etats-Unis) dans le but de mesurer le $\mathrm{pH}$, la pression partielle en $\mathrm{CO}_{2}\left(p_{\mathrm{CO} 2}\right)$ et la pression partielle en $\mathrm{O}_{2}\left(p_{\mathrm{O} 2}\right)$. Les valeurs de bicarbonates $\left(\left[\mathrm{HCO}^{-}\right]\right)$ont été calculées à partir de l'équation de Henderson-Hasselbach. Les prélèvements pour la mesure de ces valeurs ont été effectués à différents instants : T1 : avant ingestion ; T2 : 1 h30 après ingestion (i.e. avant test); T3: fin du test de $\mathrm{VO}_{2}$ max Ergocyle ; T4 : fin du test de Tlim Course à pied ; T5 : 15 minutes après la fin de l'enchaînement E-TR.

\section{Effort perçu}

Les valeurs d'effort perçu ont été relevées à la fin de chaque palier lors du test triangulaire d'ergocycle et à la fin du test de temps limite en course à pied sur tapis roulant. L'échelle utilisée était celle de Borg (1970).

\section{Statistiques}

La passation des tests a été tirée au sort par les sujets afin de ne pas introduire un effet d'ordre. D'autre part, plusieurs phases d'apprentissage au test d'enchaînement ont été réalisées pour ne pas induire un biais de type « habileté spécifique » lors des évaluations.

La vitesse au seuil ventilatoire a été obtenue à partir d'une extrapolation des différentes valeurs encadrant cette zone de travail. Plusieurs paramètres ont été associés à cette intensité, à savoir la fréquence cardiaque et la consommation d'oxygène, mais également le débit ventilatoire.

Les valeurs représentent la moyenne \pm l'écart type à la moyenne. Une analyse de variance à deux facteurs (Situation $\times$ Performance) a été réalisée. Lorsque la différence était significative, la comparaison entre les différents indices obtenus ( $\left.\mathrm{pH},\left[\mathrm{HCO}^{-}\right],\left[\mathrm{La}^{-}\right], \mathrm{VO}_{2}, \mathrm{~V}_{\mathrm{E}}, \mathrm{FC}\right)$ a été réalisée à partir d'un test paramétrique pour des échantillons appariés $(t$ de Student). Le seuil significatif a été fixé à $p<0.05$. 


\section{Résultats}

Les résultats de ce travail ne révèlent aucune différence significative dans les valeurs de $\mathrm{VO}_{2}$ max relevées lors du test de course à pied et celui de cyclisme réalisé sur ergocycle (Situation Placebo, Tableau 1 et 2). De plus, indépendamment de la boisson ingérée, $\mathrm{VO}_{2}$ max et $\mathrm{FCmax}$ ne sont pas modifiés lors des 4 tests triangulaires effectués sur ergocyle (Tableau 2). Aucune différence significative n'est enregistrée pour les débits ventilatoires également, $\mathrm{V}_{\mathrm{E}}$. Les valeurs de puissance maximale aérobie (Pmax) atteintes lors de $\mathrm{C}-\mathrm{CHO}$ sont significativement supérieures à la situation $\mathrm{C}(p<0,05)$ et aux situations $\mathrm{CHO}$ et $\mathrm{P}(p<0,01)$. Les $\%$ de $\mathrm{VO}_{2}$ max à $\mathrm{S}_{\mathrm{V} 2}$ calculés dans les situations $\mathrm{C}$ et $\mathrm{C}-\mathrm{CHO}$ sont significativement plus élevées que dans les situations $\mathrm{CHO}$ et $\mathrm{P}(p<0,05)$.

Les valeurs d'effort perçu lors du test triangulaire ( $c f$. Figure 1) en cyclisme montrent des valeurs significativement plus faibles en condition $\mathrm{C}-\mathrm{CHO}$ comparées aux valeurs relevées dans les conditions $\mathrm{CHO}$ et $P$ à partir du palier $150 \mathrm{~W}$ et jusqu'à la fin du palier $375 \mathrm{~W}(p<0,01$ de 150 à $250 \mathrm{~W}$, et $p<0,05$ de 275 à $375 \mathrm{~W}$ ). Les valeurs sont significativement plus faibles en condition $\mathrm{C}-\mathrm{CHO}$ vs. C uniquement du palier 150 watts au palier 250 watts $(p<0,01)$, les valeurs n'étant plus significativement

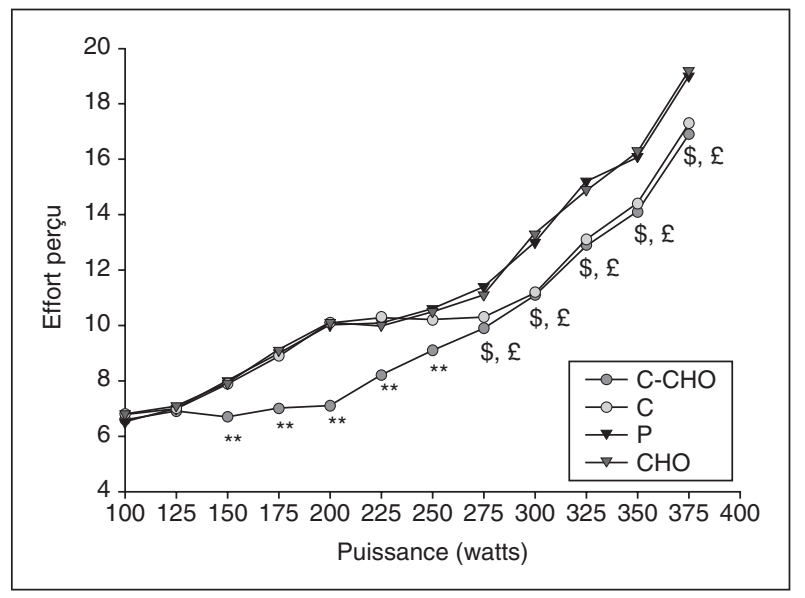

FIGURE I

Valeurs d'effort perçu obtenues lors du test triangulaire sur Ergocycle. Les valeurs indiquées sont les valeurs moyennes de la population enregistrées à la fin de chaque palier du test incrémenté. $\mathrm{C}-\mathrm{CHO}$ : Citrate + hydrates de carbone ; $\mathrm{C}$ : Citrate ; $\mathrm{CHO}$ : Hydrates de carbone ; P : Placebo. Différence significative avec les groupes $\mathrm{C}, \mathrm{P}$ et $\mathrm{CHO}$ correspondant, ${ }^{* *} p<0,0 \mathrm{I}$.

Différence significative du groupe $\mathrm{CHO}$ avec les groupes $\mathrm{C}-\mathrm{CHO}$ et $\mathrm{C}$ correspondant, $\$ p<0,05$. Différence significative du groupe $\mathrm{P}$ avec les groupes $\mathrm{C}-\mathrm{CHO}$ et $\mathrm{C}$ correspondant, $£ p<0,05$. 


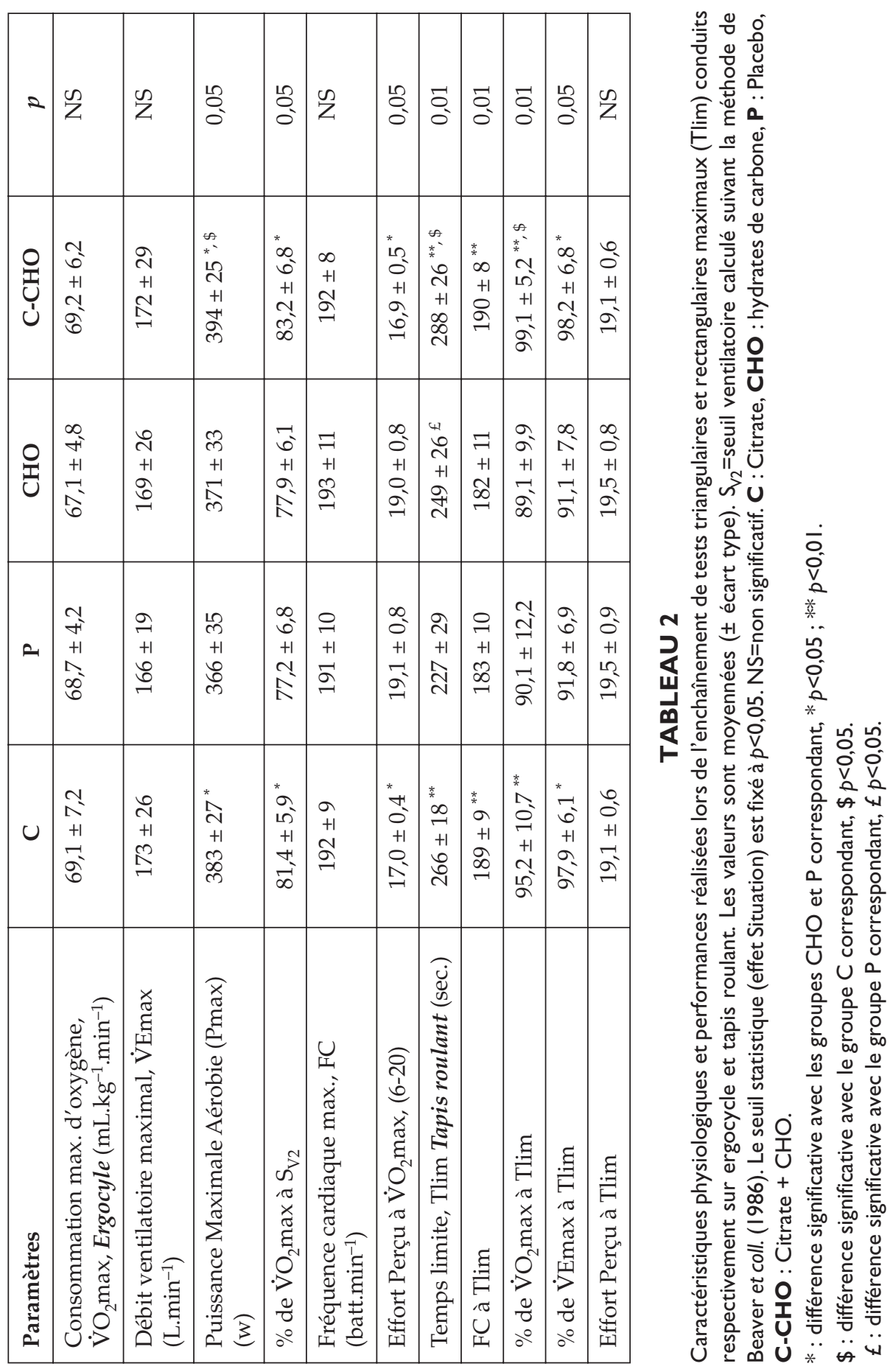


différentes ensuite, et ce jusqu'à la fin du test. Les valeurs relevées en situation $C$ sont significativement plus faibles $(p<0,05)$ que celles des conditions $\mathrm{CHO}$ et $\mathrm{P}$ du palier $275 \mathrm{~W}$ jusqu'à la fin du palier $375 \mathrm{~W}$.

Les valeurs de changements de concentrations en bicarbonates sanguins et de $\mathrm{pH}$ sanguins ne sont pas significativement différentes avant l'ingestion ( $c f$. Figure 2A et 2B). A l'instant T2 (i.e. 1 h30 après ingestion), pour $\left[\mathrm{HCO}^{-}\right]$et le $\mathrm{pH}$ les valeurs en conditions $\mathrm{C}-\mathrm{CHO}$ et $\mathrm{C}$ sont significativement plus élevées comparées aux situations $\mathrm{CHO}$ et $\mathrm{P}(p<0,01)$. Les mêmes différences sont enregistrées entre les instants T3, T4 et T5 $(p<0,01)$, à la fois pour [ $\left.\mathrm{HCO}^{-}\right]$et le $\mathrm{pH}$.

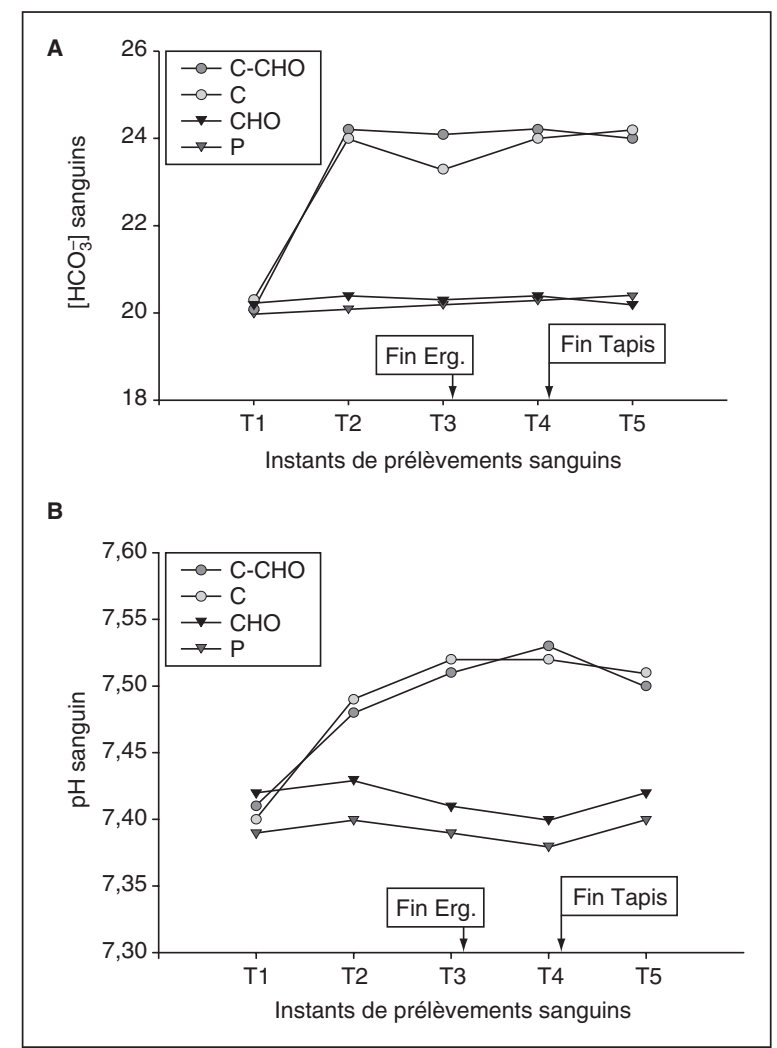

FIGURE 2

Changements de concentrations en bicarbonates sanguins (A) et $\mathrm{pH}(\mathbf{B})$ avant ingestion (TI), après ingestion (T2), après le test d'Ergocyle (T3), après le test de Tapis roulant (T4) et 15 minutes après la fin de l'enchaînement (T5). C-CHO : Citrate + hydrates de carbone; $\mathrm{C}$ : Citrate ; $\mathrm{CHO}$ : Hydrates de carbone ; P : Placebo. En T2, T3, T4 et T5, les valeurs de [HCO3] et de $\mathrm{pH}$ en $\mathrm{C}-\mathrm{CHO}$ et $\mathrm{C}$ sont significativement supérieures aux conditions $\mathrm{CHO}$ et $\mathrm{P}(p<0,0 \mathrm{I})$. 


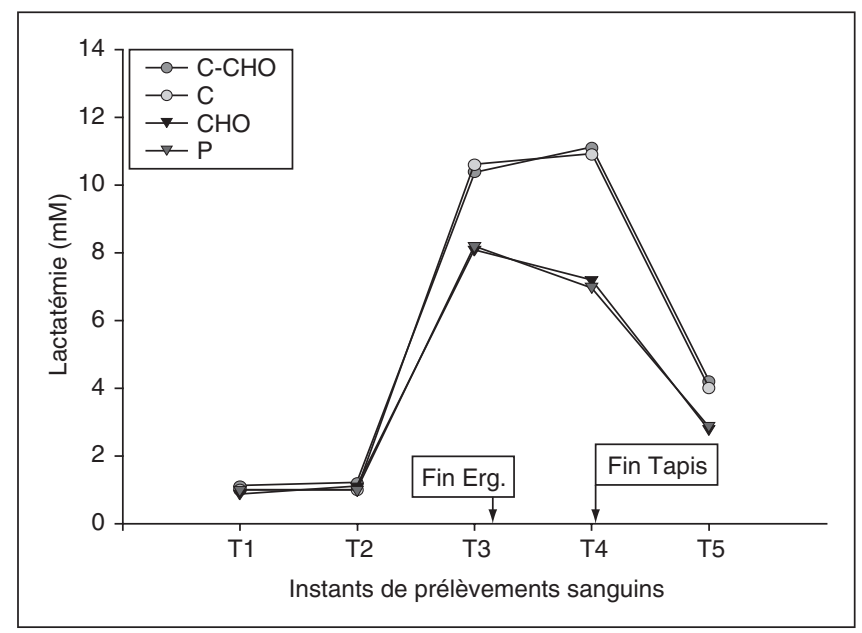

FIGURE 3

Changements de concentrations en lactates sanguins avant ingestion (TI), après ingestion (T2), après le test d'Ergocyle (T3), après le test de Tapis roulant (T4) et 15 minutes après la fin de l'enchaînement (T5). C-CHO : Citrate + hydrates de carbone ; C : Citrate ; $\mathrm{CHO}$ : Hydrates de carbone ; P : Placebo. En T3, T4 et T5, les valeurs de [La-] en $\mathrm{C}-\mathrm{CHO}$ et $\mathrm{C}$ sont significativement supérieures aux conditions $\mathrm{CHO}$ et $\mathrm{P}(p<0,0 \mathrm{I})$.

Les valeurs de lactatémies sont significativement plus élevées aux instants T3, T4 et T5 pour les conditions $\mathrm{C}-\mathrm{CHO}$ et $\mathrm{C}$ en comparaison avec les valeurs relevées dans les conditions $\mathrm{CHO}$ et $\mathrm{P}$ correspondantes (cf. Figure 3). Aucune différence significative n'a été enregistrée pour les valeurs de lactatémies avant ingestion (T1) et 1 h30 après ingestion (T2).

\section{Discussion}

Le résultat principal de cette étude est que l'ingestion d'une solution de citrate de sodium tri-sodique associée à des hydrates de carbone, des vitamines et des minéraux ( $\mathrm{C}-\mathrm{CHO})$ améliore d'une part la puissance maximale aérobie en cyclisme sans modifier toutefois la $\mathrm{VO}_{2}$ max, et d'autre part augmente la performance en terme de temps limite au second seuil ventilatoire en course à pied réalisée en enchaînement du test de cyclisme chez des triathlètes entraînés.

L'effet ergogénique d'une ingestion de bicarbonate de sodium et de citrate de sodium a été largement décrit dans la littérature pour des exercices relativement courts et intenses. Les résultats montrent un effet 
bénéfique sur des périodes de temps comprises entre 1 à 6 min en exercice global (Bouissou et coll., 1988 ; McNaughton 1990) et entre 2 et 5 min en exercice local isométrique (Hausswirth et coll., 1995 ; Maughan et coll., 1986). En revanche, aucune étude n'a étudié l'effet d'une alcalose induite sur un temps limite $\left(\mathrm{T}_{\mathrm{lim}}\right)$ réalisé après un test triangulaire mené jusqu'à épuisement. La présente étude détermine un effet positif de l'ingestion de citrate de sodium tri-sodique (C) sur la Pmax en cyclisme, en comparaison avec un placebo $(\mathrm{P} ;+5 \%)$ et une solution classique $\mathrm{d}$ 'hydrates de carbone $(\mathrm{CHO} ;+4 \%)$. Cette différence en terme de performance n'est pas attribuée à des modifications de $\mathrm{VO}_{2} \max$ ni de $\mathrm{V}_{\mathrm{E}^{\prime}}$ ces valeurs restant identiques entre les situations. La principale modification est obtenue pour le $\%$ de $\mathrm{VO}_{2}$ max à $\mathrm{S}_{\mathrm{V} 2}$, où le pourcentage en $\mathrm{C}$ est supérieur à celui calculé en $\mathrm{CHO}$ ou en $\mathrm{P}$. De ce fait, au niveau du seuil ventilatoire, une meilleure extraction périphérique de l'oxygène est rendue possible par l'alcalose, reculant ainsi les effets délétères de l'acidose métabolique de l'exercice. En effet, nous avons montré que les valeurs de bicarbonates et de $\mathrm{pH}$ sanguins sont augmentées par l'ingestion de citrate de sodium, et que ces valeurs plus élevées sont retrouvées à la fin du test de cyclisme. Â des intensités plus faibles (i.e. en dessous ou au niveau de $S_{\mathrm{V} 2}$ ), l'alcalose participe à un meilleur coût énergétique de l'exercice sous-maximal. Ces résultats sont à rapprocher de ceux obtenus par McNaughton et coll. (1999), montrant une amélioration des performances (i.e. \% de $\mathrm{VO}_{2}$ max plus élevés) lors de 60 min de cyclisme : ce meilleur rendement énergétique a été attribué à l'alcalinisation préalable, comme comme en témoignaient les valeurs de bicarbonates plasmatiques plus élevées tout au long des $60 \mathrm{~min}$. Par ailleurs, tout comme l'étude de Brisswalter et coll. (1999), une solution de CHO n'a pas d'effet ergogénique sur le coût énergétique de la course à pied chez des triathlètes entraînés. Dans notre étude, l'effort perçu est plus faible en situation $\mathrm{C}$ comparé à $\mathrm{P}$ et $\mathrm{CHO}$, mais uniquement à partir des paliers $275 \mathrm{~W}$ et jusqu'à la fin du test de cyclisme. Une étude récente de Utter et coll. (2002) montrait que les marathoniens étaient capables de courir plus vite avec une ingestion de $\mathrm{CHO}$ vs. Placebo, sans toutefois modifier l'effort perçu. Dans notre étude, $\mathrm{T}_{\text {lim }}$ est plus élevé de $9 \%$ entre $\mathrm{CHO}$ et $\mathrm{P}$, sans modification toutefois de l'effort perçu en fin de course à pied, et pour des valeurs de lactatémies similaires. La situation $C$ augmente le $\mathrm{T}_{\text {lim }}$ comparé à $\mathrm{P}(+15 \%)$ et $\mathrm{CHO}(+7 \%)$. Ce résultat est relié à des lactatémies plus élevées ainsi que des bicarbonates et $\mathrm{pH}$ sanguins augmentés. Ce résultat est souvent retrouvé dans la littérature pour des exercices de course à pied isolés : les temps de course sont améliorés par 
l'alcalinisation exogène sur des exercices de $400 \mathrm{~m}$ (Goldfinch et coll., 1988) et de $800 \mathrm{~m}$ (Wilkes et coll., 1983). Notre étude montre de plus que $\mathrm{C}$ induit des $\mathrm{T}_{\lim }$ supérieurs à une situation $\mathrm{CHO}$. L'effet ergogénique de l'alcalose est connu pour augmenter les efflux de protons et d'ions lactates en provenance du muscle, améliorant ainsi le pouvoir de tamponner ces protons dans le milieu extracellulaire (McNaughton 1990). De ce fait, dans notre étude, l'ingestion de citrate de sodium provoque une augmentation de bicarbonates et permet ainsi une meilleure extrusion des ions lactates vers le milieu circulant tout en tamponnant l'excès de protons et facilitant un meilleur $\mathrm{T}_{\text {lim }}$ en course à pied.

Lors d'exercices d'intensités comprises entre 70-85 \% de $\mathrm{VO}_{2} \max$, le glycogène musculaire est le substrat le plus important à l'exercice (Coyle et coll., 1983). Quelques études ont mis en évidence qu'une supplémentation d'une solution de $\mathrm{CHO}$ pendant l'exercice était associée à une augmentation des performances; cela a été relié à un maintien de la concentration de glucose sanguin ainsi qu'à une épargne en glycogène musculaire (Coyle et coll., 1986). Par ailleurs, quelques auteurs ont pu montrer, à l'inverse, des effets négatifs de l'absorption d'une solution CHO 45 min avant le début d'un exercice (Koivisto et coll., 1981). Ils ont observé une association entre l'élévation de la concentration de glucose sanguin et la baisse des $\mathrm{T}_{\text {lim }}$ lors d'un exercice de cyclisme. Concernant notre étude, nous observons une augmentation des $\mathrm{T}_{\text {lim }}$ en course à pied dans la condition $\mathrm{CHO}$ vs. $\mathrm{P}(+9 \%)$. Ceci est attribué à la présence de polymères de glucose dans la solution $\mathrm{CHO}$, polymères ne produisant que des réponses insuliniques plus réduites en comparaison avec du glucose seul (Flynn et coll., 1987).

La solution de $\mathrm{C}-\mathrm{CHO}$ de notre étude entraîne une augmentation de la Pmax comparée à $\mathrm{C}(+3 \%)$, à $\mathrm{CHO}(+6 \%)$ et à $\mathrm{P}(+8 \%)$; ceci n'est pas expliqué par une modification de la $\mathrm{VO}_{2} \max$, de $\mathrm{FCmax}$ ou de $\vec{V}_{\mathrm{E}}$ max, ces valeurs restant identiques dans les 4 situations en fin de test d'ergocycle. Les valeurs de lactatémies, de bicarbonates et de $\mathrm{pH}$ sanguins sont plus élevées en fin de test comparées aux conditions $\mathrm{P}$ et $\mathrm{CHO}$, expliquant largement les possibles améliorations de la Pmax, comme en témoignent également les valeurs significativement plus faibles d'effort perçu durant toute la durée du test triangulaire en condition C-CHO. Pour expliquer les différences de Pmax entre $\mathrm{C}$ et $\mathrm{C}-\mathrm{CHO}$, seules les valeurs d'effort perçu peuvent rendre compte partiellement des différences, essentiellement aux intensités faibles (150 à $250 \mathrm{~W})$; la fatigue subjective ressentie par les triathlètes est plus faible en condition $\mathrm{C}-\mathrm{CHO}$, pour des intensités en dessous de $\mathrm{S}_{\mathrm{V} 2}$. N'ayant pas mesurés de 
paramètres sanguins supplémentaires ou électromyographiques (EMG) pendant l'exercice incrémenté, seule la plus faible fatigue perçue par les sujets aux intensités sous-maximales peuvent expliquer partiellement les modifications de Pmax en fin de test.

De nombreuses études ont mis en évidence des augmentations du temps limite de course à pied après ingestion de bicarbonate de sodium (voir : Hooker et coll., 1987), mais ces études souvent associées à des problèmes d'ordre méthodologique ; en effet, l'allure de course est souvent fixée à priori et les athlètes ne peuvent pas tirer profit de l'augmentation de la capacité tampon fournie. De ce fait, nous avons proposé un exercice de $\mathrm{T}_{\text {lim }}$ précédé d'un exercice épuisant de $\mathrm{VO}_{2}$ max au préalable afin que les sujets tirent partie complètement de l'augmentation du pouvoir tampon. Heigenhauser \& Jones (1991) nous indiquent que la façon dont le citrate de sodium va agir est dépendante de la capacité de travail produite. Son action sera d'autant plus efficace que l'intensité sera élevée. Les Tlim observés dans notre étude sont supérieurs en condition C$\mathrm{CHO}$ et $\mathrm{C}$, comparés aux conditions $\mathrm{CHO}$ et $\mathrm{P}$. Ceci confirme le fait que l'exercice était suffisamment épuisant pour que la capacité du pouvoir tampon lié à l'ingestion de citrate de sodium soit active. En effet, les valeurs de $\mathrm{pH}$, de bicarbonates et de lactates sanguins sont plus élevés en fin de $\mathrm{T}_{\text {lim }}$ pour ces deux situations alcalinisantes. Ces résultats sont souvent expliqués par le fait que des taux élevés de la glycolyse diminuent le $\mathrm{pH}$ intramusculaire, lui-même faisant chuter l'efficacité de contraction des protéines contractiles (Bryant-Chase \& Kushmeric, 1988). Ces auteurs ont montré que la relation est réversible, c'est-à-dire que la remise à un niveau physiologique de base ou au dessus du $\mathrm{pH}$ intramusculaire avait pour conséquence une production de force plus importante. Les meilleurs Tlim obtenus en $\mathrm{C}-\mathrm{CHO}$ et $\mathrm{C}$ dans notre étude sont à relier également aux valeurs de FC plus importantes, aux \% de $\mathrm{VO}_{2}$ max et aux \% de $\mathrm{V}_{\mathrm{E}}$ max en fin d'exercice. Ceci indique largement la faculté des sujets à pouvoir mobiliser quasi $100 \%$ de $\mathrm{VO}_{2}$ max dans la condition $\mathrm{C}-\mathrm{CHO}$, associée de ce fait à une plus grande fréquence cardiaque (i.e. débit cardiaque) en fin de test. Les différences de $\mathrm{T}_{\text {lim }}$ obtenues entre $\mathrm{C}-\mathrm{CHO}$ et $\mathrm{C}$ peuvent être expliquées par le fait que l'enchâ̂nement E-TR dure environ 45-50 min avec l'échauffement. Par conséquent, seule la solution avec polymères de glucose, vitamines et minéraux était susceptible de pouvoir jouer un rôle encore plus bénéfique sur le $\mathrm{T}_{\text {lim' }}$, performance associée à un plus faible $\%$ de $\mathrm{VO}_{2}$ max en condition $\mathrm{C}$ vs. C-CHO. Si toutefois nous avons obtenus quatre $\mathrm{T}_{\text {lim }}$ 
significativement différents entre eux, aucune différence significative n'a été relevée dans l'effort perçu en fin de test d'enchaînement E-TR.

En conclusion, cette étude a montré d'une part l'importance d'une ingestion de solution alcalinisante dans l'accomplissement d'une performance en cyclisme (Pmax) et en course à pied $\mathrm{T}_{\text {lim }}$ ), et d'autre part la réalisation d'une meilleure performance par ajout de $\mathrm{CHO}$ à cette alcalose induite. Les bénéfices ergogéniques sont à rapprocher des valeurs élevées de $\mathrm{pH}$ et de bicarbonates sanguins. Les niveaux de $\mathrm{pH}$ plus importants doivent autoriser un maintien des propriétés contractiles musculaires en facilitant les efflux d'ions lactates et de $\mathrm{H}^{+}$depuis les muscles actifs. Toutefois, les différences obtenues entre $\mathrm{C}-\mathrm{CHO}$ et $\mathrm{C}$ sont davantage à relier avec les différences d'effort perçu ; une analyse EMG des muscles concernés aurait permis d'avancer des hypothèses liés au type de recrutement musculaire et à son évolution dans cette caractéristique d'exercice épuisant. 


\section{Bibliographie}

BEAVER W.L, WASSERMAN K. \& WHIPP B.J. (1986) A new method for detecting anaerobic threshold by gas exchange. J Appl Physiol 60 : 2020-2027.

Borg G.A.V. (1970) Perceived exertion as an indicator of somatic stress. Scand J Rehab Med 2 : 92-98.

Bouissou P., Defer G., GueZENnEC C.Y., Estrade P.Y. \& SERRURIER B. (1988) Metabolic and blood catecholamine responses to exercise during alkalosis. Med Sci Sports Exerc $20: 228-232$.

Brisswalter J., Hausswirth C., Vercruyssen F., Collardeau M., Vallier, BRYANT-CHASE P. \& KUSHMERICK M.J (1988) Effects of pH on contraction of rabbit fast and slow skeletal muscle fibers. Biophysics J 53 : 935-946. Eur J Appl Physiol 81 : 108-113.

Coyle E., Hagberg J., Hurley B., Martin W. \& Holloszy J. (1983) Carbohydrate feeding during prolonged strenuous exercise can delay fatigue. J Appl Physiol 55-230-235.

Coyle E., CogGan A., Hemmert M. \& IVy J. (1986). Muscle glycogen utilization during prolonged exercise when fed carbohydrates. J Appl Physiol 61-165-172.

FlynN M., Costill D., Hawley J., FinK W., Neufer P., Fielding R. \& SleEerer M. (1987) Influence of selected carbohydrate drinks on cycling performance and glycogen use. Med Sci Sports Exerc $19: 37-40$.

GOLDFINCH J., MCNAUGHTON L. \& DAVIES P. (1988) Induced metabolic alkalosis and its effects on 400-m racing time. Eur J Appl Physiol $57: 45-48$.

HAusswirTh C., Bigard A.X., LePERS R., BERHELOT M. \& GuEZENNEC C.Y. (1995). Sodium citrate ingestion and muscle perfomance in acute hypobaric hypoxia. Eur J Appl Physiol, 71, 362-368.

Hausswirth C., Bigard A.X., Berthelot M., Thomaidis M., GuezenneC C.Y. (1996). Variability in energy cost of running at the end of a triathlon and a marathon. Int J Sports Med, 18, 330-339.

Hausswirth C., Bigard A.X. \& Le CheVALIER J.M. (1997). The Cosmed K4 telemetry system as an accurate device for oxygen uptake measurements during exercise. Int J Sports Med, 18, 449-453.

HAUSSWIRTH C. \& BRISSWALTER J. (1999). Le coût énergétique de la course à pied de durée prolongée : étude des paramètres d'influence. Science \& Sports, 14, 5970.

HeIGENHAUSER G.J.F. \& JONES N.L. (1991) Bicarbonate loading. In : Lamb DR Williams $M H$ (Eds) Perspectives in exercise science and sports medicine, (Vol.4), ergonenics enhancement of exercise and sport. Wm.C. Brown, Dubuque, IA pp 183-212.

HERMANSEN L. \& OSNES B. (1972) Blood and muscle pH after maximal exercise in man. J Appl Physiol 32-304-308.

HOOKER S., MORGAN C. \& Wells C. (1987) Effect of sodium bicarbonate ingestion on time to exhaustion and blood lactate of $10 \mathrm{~km}$ runners. Med Sci Sports Exerc 19 :S67. 
Howley E.T., BASSET D.R. \& WelCH H.G. (1995) Criteria for maximal oxygen uptake : review and commentary. Med Sci Sport Exerc 27 : 1292-1301.

Kindermann W., Keul J. \& Huber G. (1977) Physical exercise after induced alkalosis (bicrabonate and Tris-buffer. Eur J Appl Physiol 37 :197-204.

KoIVISTO V., KARONEN S. \& NiKKILA E. (1981) Carbohydrate ingestion before exercise : comparison of glucose, fructose, and sweet placebo. J Appl Physiol $51: 783-787$.

KOWALCHUCK J.M., HeIGENHAUSER G.I.F. \& JONES N.L. (1984) Effect of pH on metabolic and cardiorespiratory responses during progressive exercise. J Appl Physiol $57: 1558-1563$.

LINDERMAN J.K. \& GOSSELINK K.L. (1994) The effects of sodium bicarbonate ingestion on exercise performance. Sports Med $18: 75-80$.

MAUGHAN R.J., LEIPER J.B. \& LiTCHFIELD P.E. (1986) The effects of induced acidosis and alkalosis on isometric endurance capacity in man exercise physiology. In : Dotson CO, Humphrey JH (Eds) Current Selected Research, vol. 2, AMS Press pp. 76-82.

MCNAUGHTON L.R. (1990) Sodium citrate and anaerobic performance : implications of dosage. Eur J Appl Physiol 61 :392-397.

MCNAughton L.R., DALTON B. \& PALMER G. (1999) Sodium bicarbonate can be used as an ergogenic aid in high-intensity, competitive cycle ergometry of $1 \mathrm{~h}$ duration. Eur J Appl Physiol 80 : 64-69.

SAHLIN K. (1978) Intracellular $\mathrm{pH}$ and bicarbonate concentration in human muscle during recovery from exercise. J Appl Physiol 45-474-480.

SWANK A. \& ROBERTSON R.J. (1989) Effect of induced alkalosis on perception of exertion during intermittent exercise. J Appl Physiol 67 : 1862-1867.

SUTTON J.R., JONES N.L. \& TOEWS C.J. (1981) Effect of pH on muscle glycolysis during exercise. Clin Sci 61-331-338.

Utter A.C., Kang J., Robertson R.J., Nieman D.C., Chaloupka E.C., SuminSKi R.R. \& PICCINI C.R. (2002) Effect of carbohydrate ingestion on ratings of perceived exertion during a marathon. Med Sci Sports Exerc $34: 1779-1784$.

WiLKES D., GLEHILL N. \& SMYTH R. (1983) Effect of acute induced metabolic alkalosis on $800 \mathrm{~m}$ racing time. Med Sci Sports Exerc 15 :277-282. 